\title{
Erratum to: Bayesian inference applied to spatio-temporal reconstruction of flows around a NACA0012 airfoil
}

\author{
Romain Leroux $^{1} \cdot$ Ludovic Chatellier $^{2,3} \cdot$ Laurent David $^{2,3}$
}

Published online: 14 December 2015

(C) Springer-Verlag Berlin Heidelberg 2015

\section{Erratum to: Exp Fluids (2014) 55:1699 \\ DOI 10.1007/s00348-014-1699-3}

In the original publication, the first author's first and last names were reversed. The correct name should be Romain Leroux. The original article has been updated accordingly.

The online version of the original article can be found under doi:10.1007/s00348-014-1699-3.

Romain Leroux

Romain.Leroux@irit.fr

Ludovic Chatellier

Ludovic.Chatellier@univ-poitiers.fr

Laurent David

Laurent.David@univ-poitiers.fr

1 Université Paul Sabatier, IRIT - Equipe ADRIA,

118 route de Narbonne, 31062 Toulouse Cedex 9, France

2 Institut P', CNRS-Universite de Poitiers-ENSMA, UPR 3346, Poitiers, France

3 Departement Fluides, Thermique, Combustion, SP2MI-Teleport 2, Boulevard Marie et Pierre Curie, BP 30179, 86962 Futuroscope Cedex, France 